\title{
Towards the development of a SiPM-based camera for the Cherenkov Telescope Array
}

\author{
G. Ambrosi ${ }^{1}$, E. Bissaldi ${ }^{2,3, a}$, L. Di Venere ${ }^{3,4}$, E. Fiandrini ${ }^{1}$, N. Giglietto ${ }^{2,3}$, F. Giordano ${ }^{3,4}$, \\ M. Ionica ${ }^{1}$, R. Paoletti ${ }^{5,6}$, D. Simone ${ }^{3}$, and V. Vagelli ${ }^{1}$ for the CTA Consortium ${ }^{7}$ \\ ${ }^{1}$ INFN - Sezione di Perugia, Perugia, Italy \\ ${ }^{2}$ Politecnico di Bari, Bari, Italy \\ ${ }^{3}$ INFN - Sezione di Bari, Bari, Italy \\ ${ }^{4}$ Università di Bari, Bari, Italy \\ 5 INFN - Sezione di Pisa, Pisa, Italy \\ ${ }^{6}$ Università di Siena, Siena, Italy \\ ${ }^{7}$ See www.cta-observatory. org for full author \& affiliation list
}

\begin{abstract}
The Italian National Institute for Nuclear Physics (INFN) is involved in the development of a prototype for a camera based on Silicon Photomultipliers (SiPMs) for the Cherenkov Telescope Array (CTA), a new generation of telescopes for ground-based gamma-ray astronomy. In this framework, an R\&D program within the 'Progetto Premiale TElescopi CHErenkov made in Italy (TECHE.it)' for the development of SiPMs suitable for Cherenkov light detection in the Near-Ultraviolet (NUV) has been carried out. The developed device is a NUV High-Density (NUV-HD) SiPM based on a micro cell of $30 \mu \mathrm{m} \times 30 \mu \mathrm{m}$ and an area of $6 \mathrm{~mm} \times 6 \mathrm{~mm}$, produced by Fondazione Bruno Kessler (FBK). A full characterization of the single NUV-HD SiPM will be presented. A matrix of $8 \times 8$ single NUV-HD SiPMs will be part of the focal plane of the SchwarzschildCouder Telescope prototype (pSCT) for CTA. An update on recent tests on the detectors arranged in this matrix configuration and on the front-end electronics will be given.
\end{abstract}

\section{Characterization of the NUV-HD SiPM}

A first extensive test campaign was conducted at the end of 2015 at the INFN laboratory of the Physics Department of Bari and it was devoted to the study of the NUV-HD SiPM produced by FBK [1] with pixel size of $30 \times 30 \mu \mathrm{m}^{2}$ and with area of $6 \times 6 \mathrm{~mm}^{2}$, in single configuration. All tests were performed in a dark box at room temperature (i.e., at $25{ }^{\circ} \mathrm{C}$ ). In order to characterize the SiPM, we illuminated it with laser LEDs at $300 \mathrm{~nm}, 345 \mathrm{~nm}, 380 \mathrm{~nm}$ and $460 \mathrm{~nm}$. The current signal was converted to a voltage signal by the op-amp based trans-impedance amplifier AdvanSiD [2], which includes a polezero compensation network. We obtained the distribution of signal events by analyzing the aquired waveforms in terms of amplitude and in terms of charge. Two distributions for $8 \mathrm{~V}$ of overvoltage $(\mathrm{OV})$ are shown in figure 1. The left panel displays the histogram of the maximum amplitude of each waveform, while the right panel shows the histogram obtained by integrating the waveform from the onset of the signal up to a certain integration time $t_{\text {int }}$. Here we chose $t_{\text {int }}=75 \mathrm{~ns}$. We then performed

\footnotetext{
a speaker, e-mail: elisabetta.bissaldi@ba.infn.it
} 

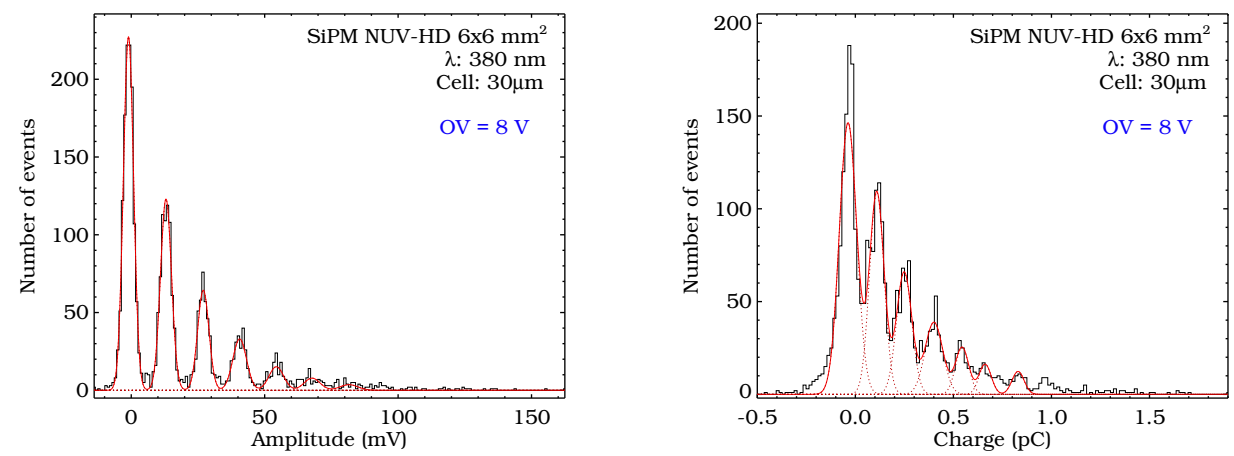

Figure 1. Distribution of signal events of the $6 \times 6 \mathrm{~mm}^{2} \mathrm{NUV}-\mathrm{HD}$ SiPM illuminated by a $380 \mathrm{~nm}$ LED and with $\mathrm{OV}=8 \mathrm{~V}$, expressed in terms of amplitude (left panel) and in terms of charge (right panel). The superimposed red line represents a Multi-Gaussian fit to the first seven photoelectron peaks.

a systematic study of the SiPM gain and of the first peak signal-to-noise ratio $\left(S N R_{1}\right)$ as a function of the integration time. Looking at the results shown in the left panel of figure 2, we can conclude that integration times in the interval $50 \mathrm{~ns}-75 \mathrm{~ns}$ represent a good compromise for obtaining high gains with a good SNR. Moreover, our tests reveal a uniformity in the behavior of the device in terms of gain. The right panel of figure 2 shows the gains versus OV calculated for different integration times and using all LEDs. The average of the slopes obtained for each wavelength is the gain $g$, expressed in $\mathrm{fC} / \mathrm{V}$. Small deviations among wavelengths are seen for long integration times and high biases. This effect is probably due to the fluctuations in the waveform tail.
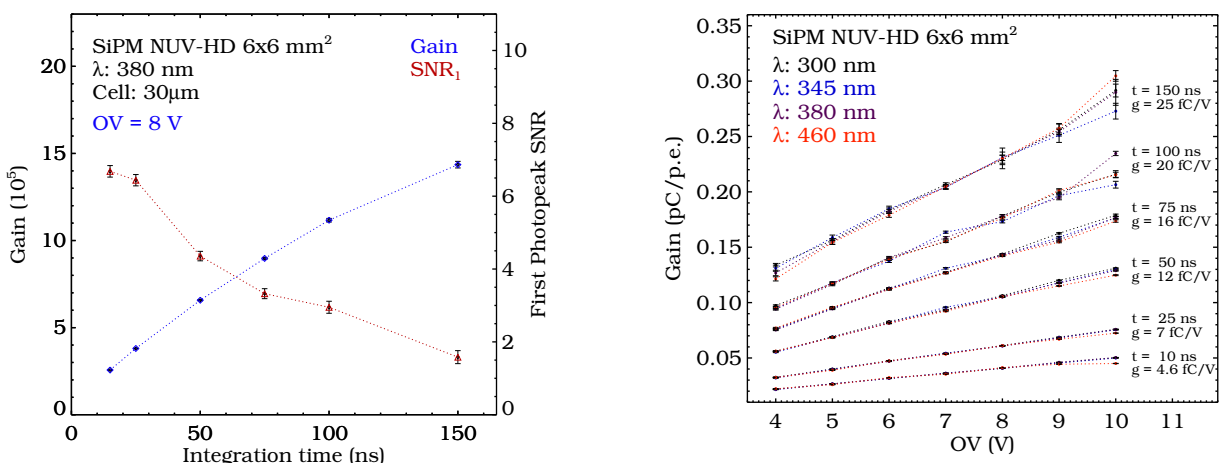

Figure 2. Left panel: SiPM gains (blue dots) and $\mathrm{SNR}_{1}$ (red triangles) of the $6 \times 6 \mathrm{~mm}^{2} \mathrm{NUV}-\mathrm{HD}$ SiPM illuminated by a $380 \mathrm{~nm}$ LED and with $\mathrm{OV}=8 \mathrm{~V}$, displayed as a function of the integration time. The connecting lines do not represent fits and just connect subsequent points. Right panel: Gain versus OV, calculated considering different integration times and using four different LEDs. 


\section{Dark count rate analysis}

In order to study the dark count rate, we performed a second set of test campaigns, which were conducted in a climate chamber located at the SITAEL laboratory [3]. The chamber was operated in complete darkness between $-40{ }^{\circ} \mathrm{C}$ and $+40{ }^{\circ} \mathrm{C}$. The waveform analysis was performed first by filtering the signal and then by applying the differential leading edge discriminator (DLED) method [4]. In this way we obtained a baseline-compensated signal by calculating the difference between the measured signal and its delayed replica. We chose a delay $\Delta \mathrm{t}=560 \mathrm{ps}$. The DLED analysis for $\mathrm{T}=0{ }^{\circ} \mathrm{C}$ and $\mathrm{OV}=6 \mathrm{~V}$ is shown in the top left panel of figure 3 . We then selected those filtered peaks which rise above a threshold set at about half of the single dark photoelectron (PE) height. In the top right panel of figure 3 we show the density plot of the obtained peak distribution versus the time distance between successive peaks. The horizontal distributions correspond to the first, second, third, etc. dark PEs (from bottom to top). A histogram of the dark PE distribution is given in the bottom left panel of figure 3. The mean time between single dark PEs is about $2 \mu$ s (see bottom right panel of figure 3), which is negligible with respect to the single PE rates due to the night sky background. In order to perform a full characterization of the forward and reverse bias, we also tested
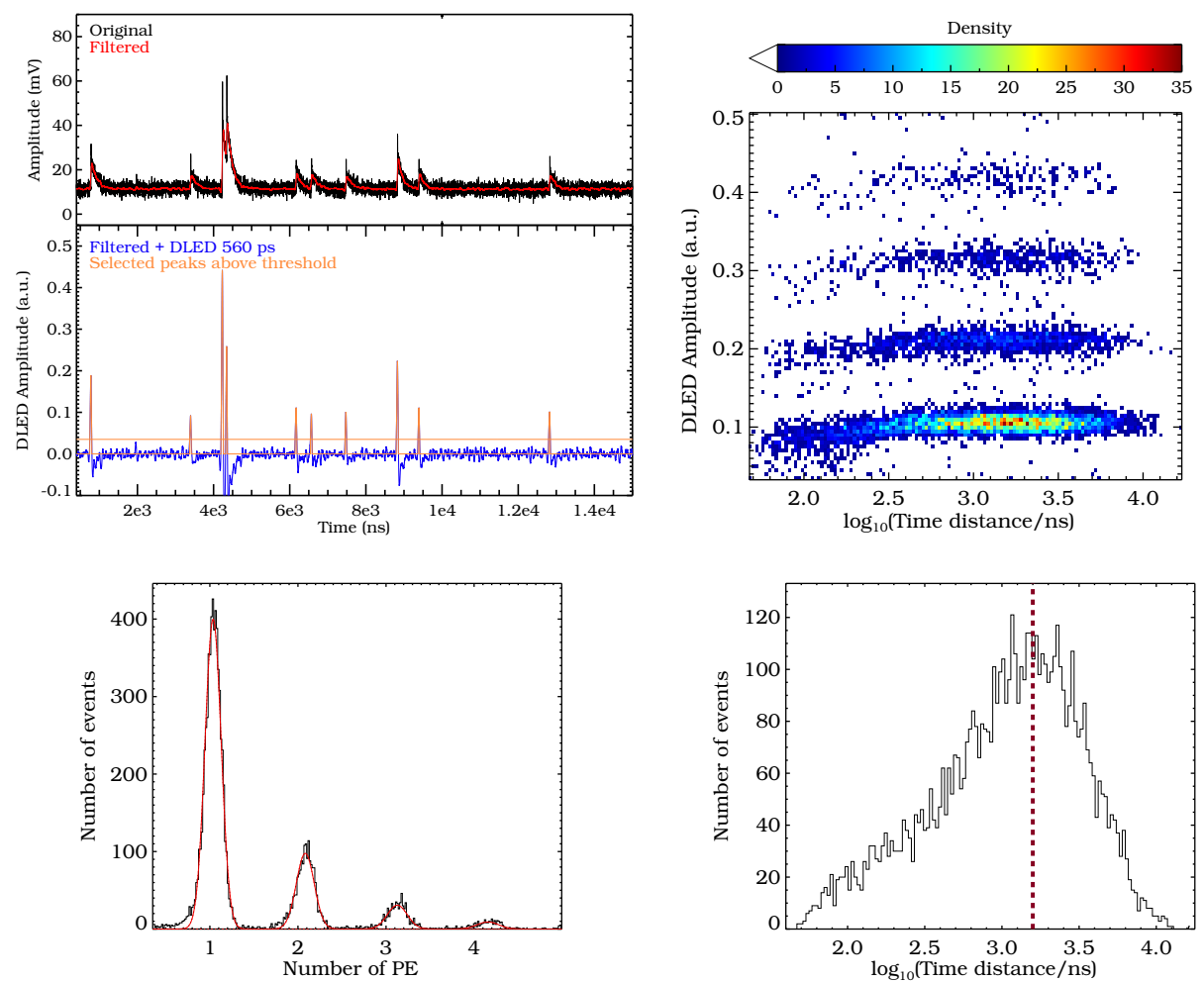

Figure 3. Top left panel: Waveform analysis of the dark signal for $\mathrm{T}=0{ }^{\circ} \mathrm{C}$ and $\mathrm{OV}=6 \mathrm{~V}$ before (top) and after (bottom) the application of the DLED method with a delay $\Delta \mathrm{t}=560 \mathrm{ps}$. Top right panel: Density plot of the peak distribution versus the time distance between successive peaks. Bottom left panel: Histogram of the dark PE distribution. Bottom right panel: Temporal distribution of the single dark PEs. The dotted red line indicates a mean value of about $2 \mu \mathrm{s}$. 

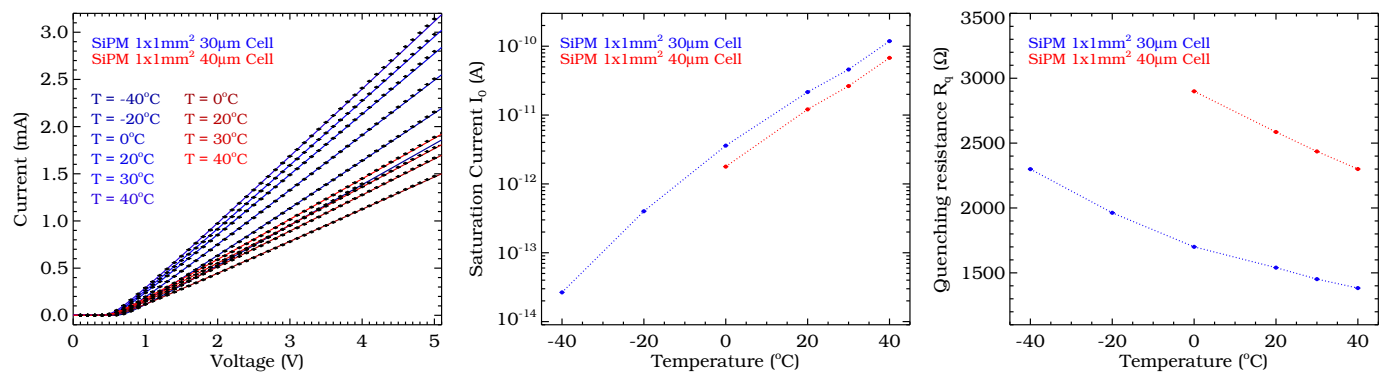

Figure 4. Forward IV-characteristics of $1 \times 1 \mathrm{~mm}^{2}$ SiPMs with $30 \mu \mathrm{m}$ (blue curves) and $40 \mu \mathrm{m}$ (red curves) cell sizes. Saturation current (middle panel) and quenching resistance (last panel) versus temperature (same color code).

in the climate chamber some SiPMs with a smaller area $\left(1 \times 1 \mathrm{~mm}^{2}\right)$ and two different cell sizes (30 $\mu \mathrm{m}$ and $40 \mu \mathrm{m})$. We derived the forward IV-characteristics, which are shown in the first panel of figure 4. From the data fitting we deduced the evolution of the saturation current (middle panel) and of the quenching resistance (last panel) as a function of the temperature for both devices (blue and red curves). This represents a crucial step towards a correct modeling of the SiPM equivalent circuit, in order to optimize the preamplifier design, i.e. getting a short signal within $10 \mathrm{~ns}$.

\section{SiPM in matrix configuration}

Finally, we performed an analysis of our NUV-HD SiPMs arranged in an $8 \times 8$ matrix configuration. We tested the reverse IV-characteristics of all 64 matrix channels and demonstrated that the breakdown voltages have a uniform behavior [5]. Moreover, we measured waveforms at different OVs by covering all but one channel with a black mask. We found that the SNRs vary between 1.5 and 5.5, whereas amplitudes are characterized by a variation of a few percent. It is interesting to note that the amplitude is generally lower if compared with the previous technology of NUV SiPMs [6]. This fact makes the NUV-HD SiPMs with $6 \times 6 \mathrm{~mm}^{2}$ area suitable detectors for the pSCT, if coupled to electronics able to detect and process positive current signals of a few millivolts per photoelectron.

\section{Acknowledgments}

We gratefully acknowledge support from the agencies and organizations listed under Funding Agencies at this website: http://www.cta-observatory.org/. E.B. ackowledges the Italian "Fondo di Sviluppo e Coesione 2007-2013 - APQ Ricerca Regione Puglia - Future In Research".

\section{References}

[1] G. Zappalà et al., JINST 11 (2016)

[2] http://advansid.com/products/product-detail/asd-ep-eb-n

[3] http://www.sitael.com

[4] A. Gola et al., IEEE Transactions on Nuclear Science 59 Issue:2 (2012)

[5] G. Ambrosi et al., Nuclear Physics B - Proceedings Supplements (CRIS2016) (2016)

[6] G. Ambrosi et al., Proceedings of Science (ICRC2015) 992 (2015) 